\title{
The Protective Effect of Taurine on the Biomembrane against Damage Produced by Oxygen Radicals
}

\author{
Tomomi Nakamura,* Mizuho Ogasawara, Ikuo Koyama, Masami Nemoto, and \\ Tsuguchika YosHIDA
}

Research Center, Taisho Pharmaceutical Co., Ltd., 1-403 Yoshino-cho, Omiya, Saitama 330, Japan. Received March 10, 1993

\begin{abstract}
The effect of taurine in protecting biomembrane against oxygen radicals was investigated using canine erythrocytes. 2,2'-Azobis(2-amidinopropane) dihydrochloride (AAPH), a water-soluble azo-compound, was used as the oxygen-radical generator. Taurine suppressed erythrocyte hemolysis more effectively than $\alpha$-alanine, used for comparison. To clarify the relationship to the lipid peroxidation, the amount of lipid peroxide was measured using liposomes prepared with egg yolk lecithin. However, the peroxidation was not suppressed by taurine. When intact erythrocytes were subjected to hemolysis by hyposmotic solutions, taurine suppressed the osmotic hemolysis more effectively than $\alpha$-alanine. These results suggest that taurine does not have an antioxidative effect like vitamin $E$, but interacts with the biomembrane, and helps to protect it against damage caused by AAPH.
\end{abstract}

Keywords taurine: biomembrane: oxidative damage; erythrocyte; oxygen radical; peroxidation

Taurine is an amino acid which is present in high concentrations in excitative cells or tissues which are susceptible to oxidation, such as brain, nerve tissues, heart, retina, neutrophils and blood platelets. ${ }^{1,2)}$ Because its physiological functions are various, there have been several reports describing the antioxidative effect of taurine against oxygen radicals. The action of taurine in combatting oxidative damage is well known to result its ability to scavenge hypochlorous acid $(\mathrm{HOCl})$ generated in neutrophils. Taurine reacts with excess $\mathrm{HOCl}$ produced in the process of phagocytosis, to form the relatively harmless monochlorotaurine. This phenomenon has been reported as being the main mechanism of the protective effect of taurine on neutrophils. ${ }^{1,3,4)}$ We have investigated the action of taurine on the oxidative damage to erythrocyte and liposomal membranes caused by $\mathrm{HOCl}$, and shown that taurine exerts a protective effect on the biomembranes against oxidative damage by trapping $\mathrm{HOCl}{ }^{5}$ )

However, when damage is caused by another oxygen radical, the role of taurine is not clear. Morales et al. ${ }^{6}$ ) reported that taurine protected frog rod outer segments from lipid peroxidation and degeneration induced by light. Banks et al. $^{7)}$ reported that incubation of alveolar macrophages with taurine before ozone exposure enhanced the viability of the cells, inhibited oxidative damage during metabolic changes such as lipid peroxidation, and suppressed leakage of intracellular glutathione; this was found to be dependent on the extracellular taurine concentration. These results suggest that taurine suppresses peroxidation induced by the oxygen radicals. Further, Morales et $a l .{ }^{8)}$ reported that taurine suppressed the accumulation of calcium and enhanced the protective action on lymphoblastoid viability, but did not inhibit lipid peroxidation. From these results they suggested that taurine acts to protect biomembranes from oxidative damage by another factor, such as control of ion permeability, and not by a direct antioxidative action. Thus, taurine behavior is influenced by both the nature of the oxygen radicals and the method used to estimate oxidation.

When oxidative damage is induced in vitro, various radical generators are used, such as light, ozone, and iron-ascorbate.
Of these radicals, Niki et $a .^{9)}$ frequently used the water soluble azo-compound, 2,2'-azobis(2-amidinopropane)dihydrochloride (AAPH), because the amount of free radicals generated can be easily controlled by adjusting the concentration of AAPH and the temperature.

In this study, to examine the protective effect of taurine on the biomembrane, we used a simple free cell, erythrocyte, and used AAPH as the oxygen-radical generator.

\section{Materials and Methods}

Materials The taurine used in this study was a standardized product (51 A.M, No. 796) recognized by the Ministry of Welfare, which had been synthesized in our laboratory. DL- $\alpha$-Alanine ( $\alpha$-alanine), DL- $\beta$-alanine ( $\beta$-alanine), and 2,2'-azobis(2-amidinopropane)dihydrochloride (AAPH) were purchased from Wako Pure Chemical Industries. 2-Amidinoethyl phosphonic acid (ciliatine) and cholesterol (CHL) were obtained from Sigma Chemicals. Dicetyl phosphate (DCP) was purchased from Aldrich Chemicals. "Coatsome," which contained $20 \%$ egg yolk lecithin as an ethanolic solution, was purchased from Nippon Oil and Fats Co., Ltd. The other chemicals used in this study were reagent grade commercial products.

Erythrocyte Suspension Fresh blood from a beagle dog was collected in heparinized tubes. Erythrocytes were separated by centrifugation at $550 \times \mathrm{g}$, for $5 \mathrm{~min}$, at $4^{\circ} \mathrm{C}$ and washed three times with $0.9 \%$ sodium chloride solution which was adjusted to $\mathrm{pH} 7.4$ by sodium hydroxide. Then, cells were diluted with $0.9 \%$ sodium chloride solution, to give a suspension of $4 \times 10^{8}$ or $4 \times 10^{7}$ cells $/ \mathrm{ml}$, and used for the experiments.

Erythrocyte Hemolysis with AAPH Two $\mathrm{ml} 45 \mathrm{~mm}$ isosmotic amino acid solution or physiological buffered saline (PBS, pH 7.4) and $1 \mathrm{ml} 25 \mathrm{~mm}$ isosmotic AAPH ( $\mathrm{pH} \mathrm{7.4)}$ were added to $1 \mathrm{ml}$ erythrocyte suspension $\left(4 \times 10^{7}\right.$ cells $\left./ \mathrm{ml}, \mathrm{pH} 7.4\right)$. The mixture was incubated at $37^{\circ} \mathrm{C}$ to initiate the radical reaction. After a defined period of treatment, the erythrocyte suspension was centrifuged at $550 \times \mathrm{g}$, for $5 \mathrm{~min}$, at $4^{\circ} \mathrm{C}$. The supernatant was used for the estimation of hemoglobin-Fe by atomic absorption spectrometry (Hitachi, Z-6000).

Erythrocyte Hemolysis Induced by Osmotic Shock Nine $\mathrm{ml} 20 \mathrm{~mm}$ taurine or $\alpha$-alanine solution, which were isotonized by sodium chloride and adjusted to $\mathrm{pH} 7.4$ by sodium hydroxide, were added to $1 \mathrm{ml}$ erythrocyte suspension $\left(4 \times 10^{8}\right.$ cells $\left./ \mathrm{ml}\right)$. The mixture was allowed to stand for $30 \mathrm{~min}$ at $4^{\circ} \mathrm{C}$, and then portions was added to 3 volumes of solutions with different osmotic pressures (120-190 mOsm, final osmotic pressure). After $10 \mathrm{~min}$ of incubation at $37^{\circ} \mathrm{C}$, the mixture was centrifuged at $550 \times \boldsymbol{g}$, for $5 \mathrm{~min}$, at $4^{\circ} \mathrm{C}$. The supernatant was used to measure the absorbance of hemoglobin at $414 \mathrm{~nm}$ by means of an immunoreader (MCC, Titertek Murtiskan).

Estimation of Hemolytic Rate The hemolytic rate was calculated as the percent of hemolysis. The absorbance of hemoglobin or hemoglobin-Fe, 
when erythrocyte solutions were added to 3 volumes of distilled water and hemolyzed completely, was taken as being $100 \%$.

Egg Yolk Lecithin Peroxidation with AAPH A thin lipid membrane film was prepared from a chloroform solution containing $42.1 \mu \mathrm{mol}$ egg yolk lecithin (egg-PC) and $5 \mu \mathrm{mol}$ dicetyl-phosphate (DCP), and a liposomal solution was obtained by hydration with PBS ( $\mathrm{pH}$ 7.4). Then $250 \mu \mathrm{l} 40 \mathrm{~mm}$ taurine and $250 \mu \mathrm{l} 50 \mathrm{~mm}$ AAPH were added to $500 \mu \mathrm{l}$ of liposome solution. After a defined period of treatment at $37^{\circ} \mathrm{C}$, the residual egg-PC was estimated.

Estimation of Residual Egg-PC Residual egg-PC was estimated by HPLC (Hitachi, 655A). HPLC was carried out using a LiChrosorb Si60 (Merck) column. The conditions of chromatography were as follows: temperature, $25^{\circ} \mathrm{C}$; injection volume, $25 \mu \mathrm{l}$; mobile phase, $\mathrm{MeOH} / 40 \mathrm{~mm}$ $\mathrm{NaH}_{2} \mathrm{PO}_{4}(96: 4, \mathrm{v} / \mathrm{v})$; and wavelength, $210 \mathrm{~nm}$.

Preparation of Glucose-Containing Liposomes A thin lipid membrane film was prepared from a chloroform solution containing $20 \mu \mathrm{mol}$ egg-PC, $20.3 \mu \mathrm{mol}$ cholesterol and $2.7 \mu \mathrm{mol}$ DCP. After hydration with $5 \mathrm{ml} 300 \mathrm{~mm}$ glucose/PBS $(1: 9, \mathrm{v} / \mathrm{v})$, the liposomes were submitted to an extruder for sizing at $200 \mathrm{~nm}$ and dialyzed against PBS ( $\mathrm{pH}$ 7.4) to remove excess glucose.

Estimation of Glucose Leakage from the Liposomes by AAPH Fifty $\mu \mathrm{l}$ liposome suspension was added to $100 \mu \mathrm{l}$ PBS or $80 \mathrm{~mm}$ taurine solution or $80 \mathrm{~mm} \alpha$-alanine solution (pH 7.4) in micro-tubes. Fifty $\mu$ l $50 \mathrm{~mm} \mathrm{AAPH}$ was added to the above suspensions and the solutions incubated at $37^{\circ} \mathrm{C}$ for a defined period. Twenty $\mu$ l of the mixture was used to estimate glucose leakage. Determination of glucose was performed by the method of Inoue et $a l .{ }^{10)}$ The glucose leakage rate was calculated from values obtained using liposomes completely destroyed with chloroform.

\section{Results and Discussion}

As shown in Fig. 1, the erythrocyte hemolysis was increased by AAPH and was dependent on the incubation time. It is known that AAPH yields a steady supply of oxygen radicals due to their unimolecular thermal decomposition and this initiates a free-radical chain mechanism. It also oxidizes erythrocyte membranes from the outside. ${ }^{9)}$

The lag-time for the hemolysis was retarded in the presence of taurine, $\beta$-alanine, and ciliatine. The data suggest that these amino acids are involved in inhibiting the oxidative damage to the erythrocytes. However, $\alpha$-alanine did not show this suppressive action.

The amount of oxygen radicals generated by AAPH was dependent on temperature and the presence of $\mathrm{O}_{2}$. The components of the biomembranes are attacked by the oxygen radicals, and this is followed by a chain reaction of

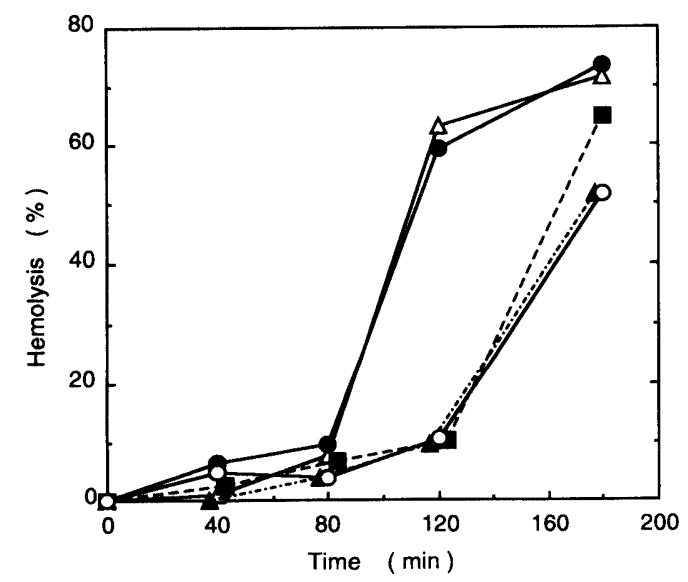

Fig. 1. Effect of Various Amino Acids on the Suppression of Hemolysis Induced by AAPH

○, PBS; $\bigcirc$, taurine; $\triangle, \alpha$-alanine: $\Delta, \beta$-alanine; $\boldsymbol{n}$, ciliatine. Erythrocytes were incubated at $37^{\circ} \mathrm{C}$ with various amino acids $(22.5 \mathrm{~mm}$, final concentration) and AAPH (6.25 mm, final concentration). lipid peroxidation. ${ }^{9)}$ To investigate that effect of taurine on lipid peroxidation, we prepared liposomes and estimated the residual egg-PC. As shown in Fig. 2, taurine did not demonstrate any suppressive effect on egg-PC peroxidation.

Also, we estimated glucose leakage from glucosecontaining liposomes induced by AAPH to examine the interaction between membrane lipids and taurine.

The glucose leakage from glucose-containing liposomes (PC: $\mathrm{CHL}: \mathrm{DCP}=4: 5: 1$ ) was increased with incubation time. However, as shown in Fig. 3a, the inhibitory effect of taurine on glucose leakage was not observed at $3 \mathrm{~h}$. So, we used another lipid composition (PC: $\mathrm{CHL}: \mathrm{DCP}=1: 0.75$ : 0.1 , reflecting the proportion of $\mathrm{PC}$ to $\mathrm{CHL}$ in the erythrocyte membrane. ${ }^{11)}$ But, again, taurine failed to suppress glucose leakage (Fig. 3b).

Takeuchi et al. ${ }^{12)}$ reported that taurine inhibited the hemolysis or the leakage of fluorescence probes from liposomes, induced by chloropromazine (CPZ). Furthermore it was shown in their experiment using electron spin resonance that taurine inhibited the penetration of $\mathrm{CPZ}$

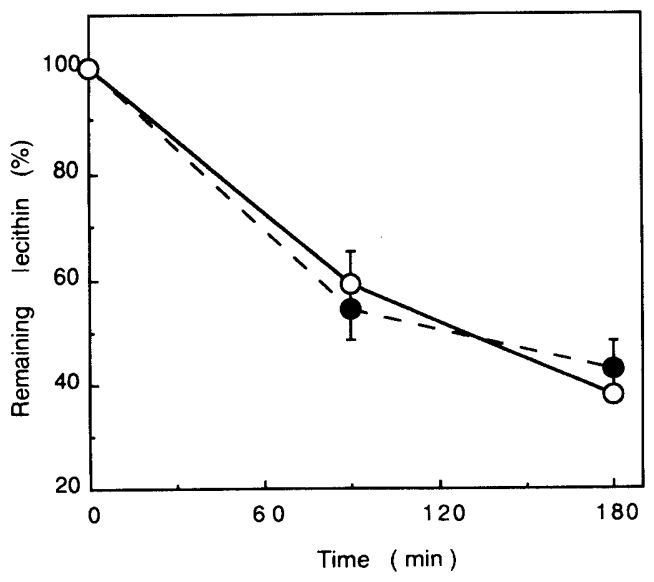

Fig. 2. Effect of Taurine on the Protection of Liposomal Lecithin against Peroxidation

, PBS; $\bigcirc, 10 \mathrm{~mm}$ taurine (final concentration). Liposomes prepared from egg-PC $(43.1 \mu \mathrm{mol})$ and DCP $(5 \mu \mathrm{mol})$ were incubated at $37^{\circ} \mathrm{C}$ with taurine and AAPH $(12.5 \mathrm{~mm}$, final concentration). Each point represents the mean \pm S.D. $(n=3)$.

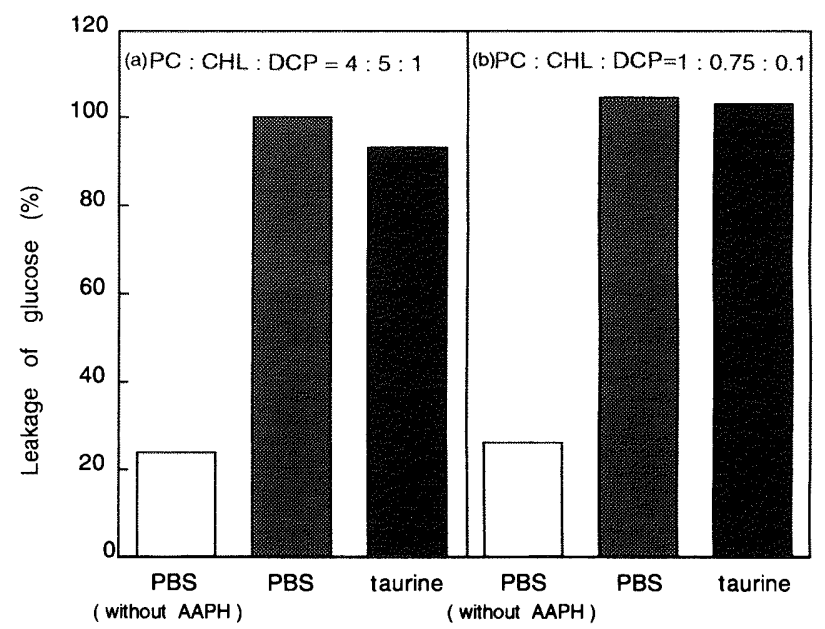

Fig. 3. Effect of Taurine on the Suppression of Glucose Leakage from Liposomes Induced by AAPH

Liposomes prepared from egg-PC, $\mathrm{CHL}$, and DCP were incubated in PBS at $37^{\circ} \mathrm{C}$, for $3 \mathrm{~h}$ with AAPH $(12.5 \mathrm{~mm}$, final concentration) in the presence or absence of taurine ( $40 \mathrm{~mm}$, final concentration). Each value represents the mean of two experiments. 


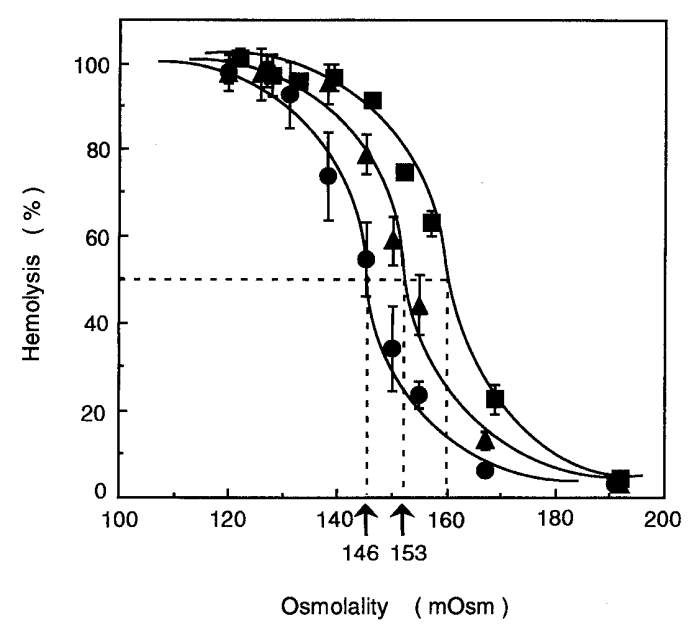

Fig. 4. Effect of Pretreatment with Taurine on the Suppression of Hemolysis Induced by Osmotic Shock

, PBS;, $4.5 \mathrm{~mm}$ taurine; $\boldsymbol{\Lambda}, 4.5 \mathrm{~mm} \alpha$-alanine (final concentration). Erythrocytes were pretreated in PBS with amino acids $\left(4^{\circ} \mathrm{C}, 30 \mathrm{~min}\right)$. After treatment the mixture was added to saline solutions of different osmolality $(120-190 \mathrm{mOsm})$. Each point represents the mean \pm S.D. $(n=4)$.

into the innermost portion of the lipid membranes. ${ }^{13)}$ They suggested that taurine inhibited the membrane perturbing action of $\mathrm{CPZ}$ by surrounding the membrane. In the case of damage by AAPH, it is known that the oxygen radicals which are generated react immediately with components of the membrane, in addition to the penetration of AAPH itself. $^{14)}$ Although taurine does not inhibit the radical reaction, it may suppress the perturbation of the membrane caused by degeneration of the membrane components.

Huxtable et al. ${ }^{15,16)}$ reported that taurine exhibits low affinity binding to cardiac sarcolemma, and similar levels of binding were observed in phospholipid vesicles, which included phospholipids in the approximate proportions reported for rat heart sarcolemma. They further reported that taurine interacted with neutral phospholipids, particularly phosphatidylcholine and phosphatidylethanolamine. Erythrocyte membranes include not only phosphatidylcholine but other phospholipids, for example phosphatidylethanolamine and phosphatidylserine. So, a similar binding effect of taurine may be observed in erythrocytes.

Since hemolysis is also induced by osmotic shock, we investigated the effect of taurine against the hemolysis caused by such shock to study the interaction between taurine and the membrane. As shown in Fig. 4, the hemolytic rate was increased as the solutions, in which the erythrocytes were suspended, became hyposmotic.

Fifty percent of erythrocytes were hemolyzed at 160 mOsm (final osmolarity) in PBS without amino acid. However, by pre-treating with taurine, $50 \%$ hemolysis took place at $146 \mathrm{mOsm}$. Thus, taurine has a remarkable effect on the suppression of the hemolysis. $\alpha$-Alanine also inhibits hemolysis, but is less effective than taurine.

These data indicate that taurine protects biomembranes from instability caused by peroxidation, not by blocking the chain radical reaction, but by interaction with the biomembranes, and suppression of hemolysis as a result.

Vitamin E exists in the erythrocyte membrane and plays a role in protecting erythrocytes from oxidative damage. ${ }^{14)}$
There have been a number of reports of the suppressive effect of vitamin $\mathrm{E}$ on hemolysis caused by AAPH. ${ }^{9,17)}$ Several reports have suggested that some amino acids have a synergistic effect on the antioxidative action of vitamin E. ${ }^{18,19)}$ So, taurine may have a similar synergistic effect on antioxidants, such as vitamin $\mathrm{E}$, in the case of the erythrocyte membrane.

We investigated membrane lipids in this study, but some proteins are also present in the erythrocyte membrane. Maturo et $a{ }^{20)}{ }^{20}$ reported interaction between taurine and insulin receptors. They suggested that taurine interacts with some proteins. So, taurine may suppress the oxidative damage of the erythrocyte membrane by interaction with membrane proteins.

The suppression of hemolysis induced by AAPH was also observed in the presence of $\beta$-alanine or ciliatine (Fig. 1). These are $\beta$-amino acids which have the same carbon chain structure as taurine but have different acidic groups. On the other hand, $\alpha$-alanine did not suppress the hemolysis by AAPH. Instead, it suppressed the hemolysis by osmotic shock, but was less effective than taurine. From these data it is estimated that the suppressive action of amino acids on hemolysis may be influenced by the position of the amino groups in the amino acid structure.

Although we have shown that taurine has a suppressive effect against erythrocyte hemolysis produced by AAPH, further studies are required to elucidate the detailed mechanisms of its actions.

\section{References}

1) C. E. Wright, H. H. Tallan, Y. Y. Lin, G. E. Gaull, Annu. Rev. Biochem., 55, 427 (1986).

2) J. G. Jacobson, L. H. Smith, Physiol. Rev., 48, 424 (1968).

3) S. J. Weiss, R. Kleiu, A. Silvka, M. Wei, J. Clin. Invest., 70, 598 (1982).

4) E. L. Thomas, M. B. Grisham, D. F. Melton, M. M. Jefferson, J. Biol. Chem., 260, 3321 (1985).

5) K. Nakamori, I. Koyama, T. Nakamura, T. Yoshida, M. Umeda, K. Inoue, Chem. Pharm. Bull., 38, 3116 (1990).

6) H. Pasantes-Morales, C. Cruz, Brain Res., 330, 154 (1985).

7) M. A. Banks, D. W. Porter, W. G. Martin, V. Castranova, J. Nutr. Biochem., 2, 308 (1991).

8) H. Pasantes-Morales, C. E. Wright, G. E. Gaull, Biochem. Pharmacol., 34, 2205 (1985).

9) M. Miki, H. Tamai, M. Mino, Y. Yamamoto, E. Niki, Arch. Biochem. Biophys., 258, 373 (1987).

10) K. Inoue, Biochem. Biophys. Acta, 339, 390 (1974).

11) Y. Takeuchi, Y. Yamaoka, Y. Morimoto, I. Kaneko, Y. Fukumori, T. Fukuda, J. Pharm. Sci., 78, 3 (1989).

12) Y. Morimoto, Y. Fukumori, T. Fukuda, Y. Takeuchi, Yakuzaigaku, 52, 166 (1992).

13) Y. Morimoto, H. Uchida, Y. Fukumori, T. Fukuda, Y. Takeuchi, Yakuzaigaku, 52, 231 (1992).

14) E. Niki, Bitamin, 63, 539 (1989).

15) L. A. Sebring, R. J. Huxtable, Biochem. Biophys. Acta, 884, 559 (1986).

16) R. J. Huxtable, "Taurine and the heart: The Phospholipid Connection," Kluwer Academic Publishers, Massachusetts, 1989, pp. $31-42$.

17) Y. Yamamoto, E. Niki, J. Eguchi, Y. Kamiya, H. Shimasaki, Biochim. Biophys. Acta, 819, 29 (1985).

18) C. Rousseau, C. Richard, R. Martin, J. Chim. Phys. Phys. Chim. Biol., 85, 145 (1988).

19) M. Sekine, K. Kamohara, "Handbook: Drug \& Cosmetic Materials," Nikko Chemicals, Tokyo, 1977, pp. 453-454.

20) J. Maturo, E. C. Kulakowski, Biochem. Pharmacol., 37, 3755 (1988). 